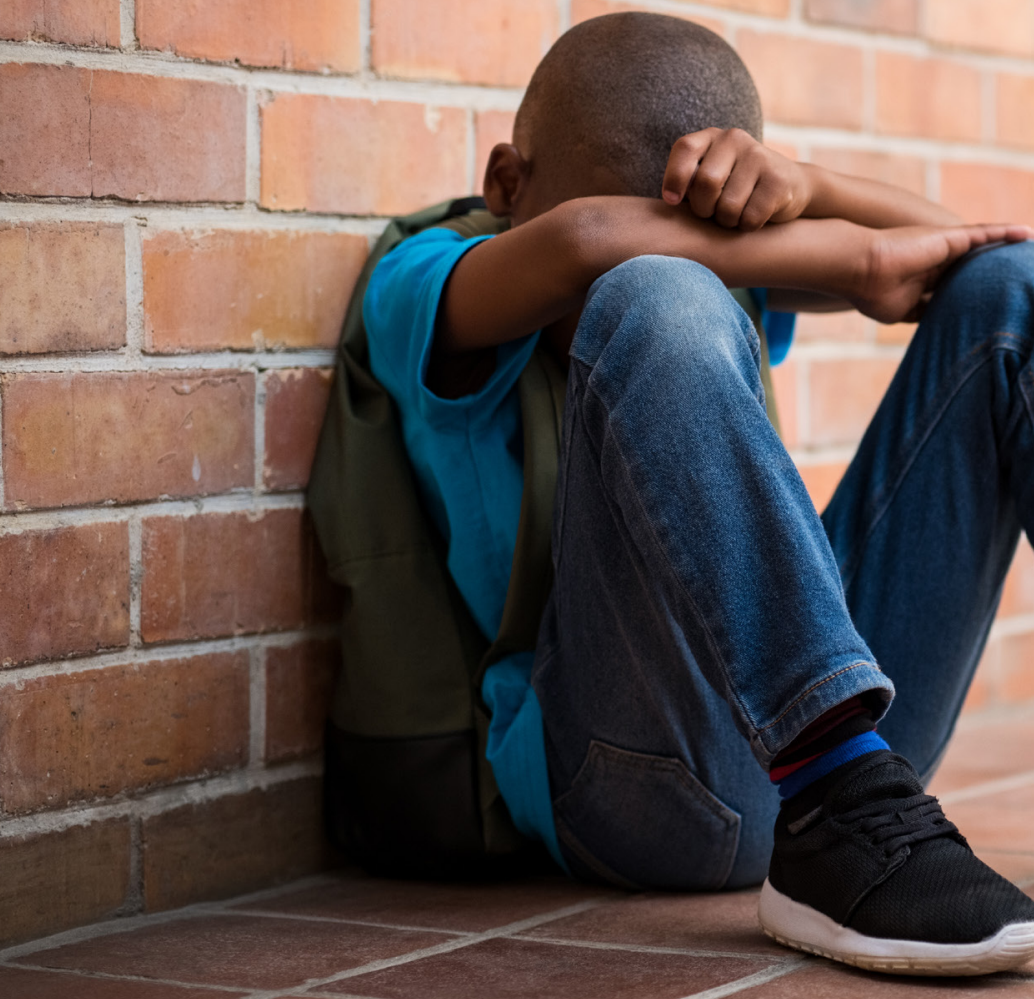

\title{
Are children of any age susceptible to suicidal behaviour?
}

By Dr. Jessica Edwards

Mei-Sing Ong and colleagues in the USA and Canada have investigated the risk factors for suicide attempt in a large cohort of children, adolescents, and young adults with mental health disorders. Ong et al. performed their cross-sectional analysis on >18,000 individuals hospitalized for a mental health disorder in a private hospital system across the USA. They found that $12.1 \%$ children (aged $\leq 12$ years) had attempted suicide 6-months before hospitalization. This percentage rose to $22 \%$ in adolescents (aged $13-17$ years) and $17.9 \%$ in young adults (aged 18-25 years).

"From these striking findings, it seems that suicidal behaviour among elementary school age children might be more pervasive than we perhaps realized", says Ong. "Historically, suicide prevention programs for youth have focused on adolescents and young adults but the data from our study show that even children as young as five can be vulnerable". Based on these data, Ong et al. urge clinicians to assume that every child with mental health concerns, regardless of age, could be at risk of suicidal behaviour.

Among other factors, including female sex and depressive disorder, the researchers found that being a victim of bullying was a major contributing factor to suicidal behaviour across all age groups. Risk factors specific to preadolescent children included having an unsafe home or school environment.

"These findings highlight the need to develop comprehensive school-based programs for preventing and addressing suicidal behaviour and bullying, and providing an environment that is sensitive to individual and societal factors that place children and youth at risk of suicidal behaviour", explains Ong. "In particular, developmentally appropriate education materials should be integrated into the curriculum of health classes to help equip children and youth with coping and help-seeking strategies."

\section{Referring to:}

Ong, M-S., Lakoma, M., Bhosrekar, S.G., Hickok, J., McLean, L., Murphey, M., Poland, R.E., Purtell, N. \& Ross-Degnan, D. (2020), Risk factors for suicide attempt in children, adolescents, and young adults hospitalized for mental health disorders. Child Adolesc. Ment. Health. doi: 10.1111/ camh. 12400 